\title{
BIOCOMBUSTIBLES PARA EL PARQUE AUTOMO- TOR DE LIMA METROPOLITANA Y EL CALLAO
}

\author{
Juvenal Cabezas Oruna
}

\section{Resumen}

En el presente artículo se revisa al comienzo la situación de calidad de los combustibles en el país al finalizar el siglo pasado, posteriormente se analiza su nocividad relacionándola con el Índice de Nocividad de Combustibles (INC) y su impacto en la contaminación del aire de la capital. A continuación se detallan las acciones tomadas especialmente en la década pasada (2000-2010) para mejorar la calidad de los combustibles como respuesta ante las críticas de la población. En dicha década coincidentemente entró en operación el proyecto Camisea el año 2005, en el mundo se hicieron más exigentes las especificaciones de calidad de los combustibles, especialmente del diesel y se empezaron a destinar áreas agrícolas en el norte del país para producir biocombustibles.

Palabras clave: GNV, GLP, INC, gasohol, biocombustibles, bioetanol, biodiesel.

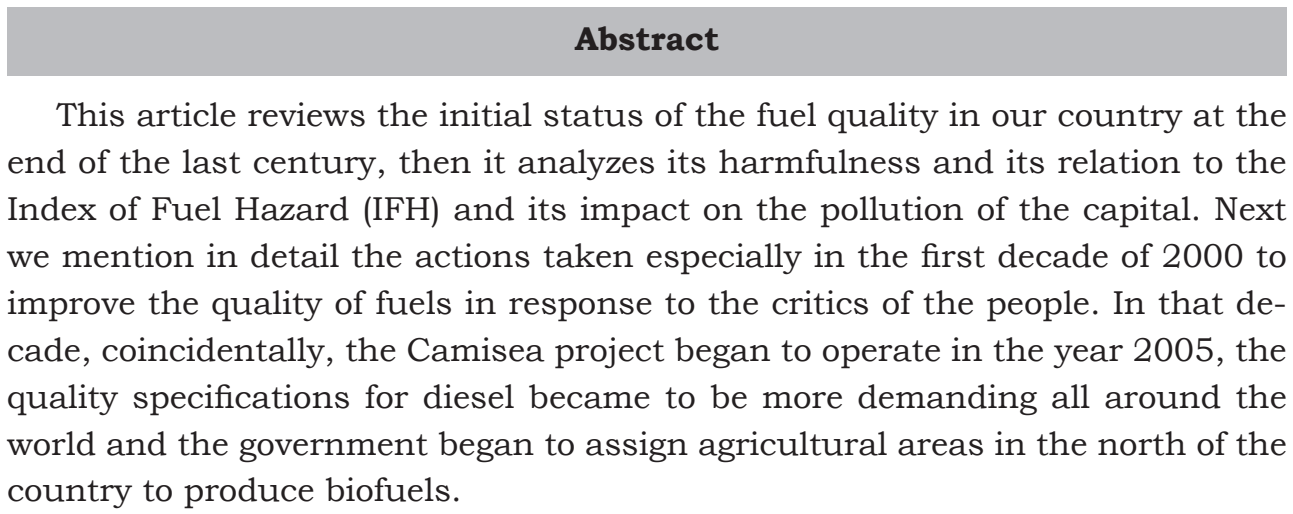

Keywords: NGV, LPG, IFH, biofuel, gasohol, bioethanol, biodiesel. 


\section{LÍNEA BASE: COMBUSTIBLES USA- DOS HASTA LA DÉCADA PASADA}

Durante el siglo pasado y hasta la primera década de este siglo, el parque automotor de Lima Metropolitana y Callao utilizó los siguientes combustibles: gasolina 84, gasolina 90, gasolina 95, gasolina 97 y diesel 2. Actualmente el parque automotor tiene a su disposición una gama variada de nuevos combustibles: gas natural vehicular (GNV), gas licuado de petróleo (GLP), gasohol 84, gasohol 90, gasohol 95, gasohol 97 y diesel B5. Tanto los gasoholes como el diesel B5 contienen biocombustibles y se comercializan desde el año 2012.

Las antiguas gasolinas y el diesel eran combustibles muy contaminantes. En el caso de las gasolinas, el contaminante principal era el plomo que se agregaba en forma de plomo tetraetílico para elevarles el octanaje. El plomo tiene la característica de que es un tóxico acumulativo bioadaptable, es decir, que puede introducirse en la cadena trófica que va del suelo a los vegetales, luego a los animales y finalmente al hombre. La intoxicación crónica es la forma más frecuente y de mayor importancia para las personas. La afectación se produce cuando los compuestos de plomo son metabolizados en el hígado; si hay un exceso de plomo, éste pasa a la circulación sanguínea y principalmente se fija en el esqueleto, dientes, médula ósea y cerebro, afectando los sistemas circulatorio, reproductor y nervioso, así como los riñones. Por eso, altos nive- les de exposición al plomo producen su bioacumulación y generan en la población encefalopatía, efectos gastrointestinales, anemia, nefropatía y anomalías electrocardiográficas; también causa retraso en el desarrollo fetal, llegando al aborto espontáneo $\mathrm{y}$, asimismo, origina la disminución de la fertilidad en los hombres. Estos problemas de salud fueron detectados en la segunda mitad del siglo pasado y la mayoría de los países cesaron el agregado de plomo tetraetílico en las gasolinas. En la década del 90, en el Perú habian dos gasolinas que no contenían plomo: las gasolinas de 90 y 97 octanos, y dos gasolinas con plomo: las de 84 y 95 octanos. En 1998, el gobierno peruano emitió el Decreto Supremo $\mathrm{N}^{\circ}$ 019-98-MTC, que en su Artículo $1^{\circ}$ prohibió la comercialización de la gasolina 95 con plomo y, desde ese mismo año, se empezó a vender sin plomo. Asimismo, dicha norma ordenó que durante los años siguientes se redujera paulatinamente el contenido de plomo tetraetílico en la gasolina de mayor venta, la de 84 octanos, terminando dicha reducción el 31 de diciembre de 2004. Es por eso que desde el $1^{\circ}$ de enero de 2005 ninguna gasolina en el Perú contiene plomo.

En el caso del Diesel, el contaminante principal ha sido el azufre. A diferencia de las gasolinas, en que se agregaba el plomo que las contaminaba, el Diesel ya contenía gran cantidad de azufre debido a que este contaminante venía con el petróleo desde el yacimiento, y la operación de destilación del petróleo solo distribuía el azu- 
fre en los diferentes productos de la destilación, entre ellos, el diesel. Adicionalmente, la norma técnica peruana se acomodaba a la calidad con que salía el diesel de las refinerías de Petroperú, las cuales no han sido modernizadas desde 1974 en que se instaló el complejo de craqueo catalítico en la refinería de Talara. El azufre originó que el diesel peruano sea uno de los combustibles automotores más sucios del planeta, con un contenido de cerca de diez mil partes por millón de azufre. Al quemarse este diesel en el motor de una unidad de transporte también se quema el azufre que contiene y se emite dióxido de azufre por el tubo de escape que, en la atmósfera, sigue oxidándose y reacciona con la humedad atmosférica produciendo micro partículas de ácido sulfúrico. Este ácido se diluye en las nubes y cae como "lluvia ácida" en la ciudad, afectando a los seres vivos, microorganismos, aguas, suelos y estructuras (restos arqueológicos, monumentos, edificios, automóviles, etc.). El ácido sulfúrico también reacciona en la atmósfera con el amoniaco, generando micro partículas de sulfato de amonio que se encuentran en el rango de tamaño de 0.1 a $1 \mu$, y que son dispersoras muy eficientes de la luz al ser parte del smog; persisten en la atmósfera hasta que coagulan y precipitan; el sulfato de amonio así generado también influye de manera significativa en los problemas urbanos de las partículas PM10. Asimismo, a nivel troposférico, el SO2 emitido por el tubo de escape y las fábricas es picante, irrita las vías respiratorias superiores (nariz, boca, garganta, etc.) y crea una tos infecciosa abundante en flema.

\section{NOCIVIDAD DE COMBUSTIBLES Y CONTAMINACIÓN DE LIMA}

Los combustibles automotores tienen diferentes características físicas y químicas que los hacen diferentes entre sí. Desde el punto de vista de contaminación ambiental su nocividad también difiere notablemente. En agosto del 2005 el entonces Consejo Nacional del Ambiente emitió el Decreto del Consejo Directivo $\mathrm{N}^{\circ}$ 018-2005-CONAM/CD que aprobaba por primera vez en el país el Índice de Nocividad de Combustibles, con la finalidad de orientar a la ciudadanía en general y a los consumidores de combustibles en particular, acerca de los diferentes tipos de combustibles según la toxicidad y nocividad ambiental que generaban. Como se observa en la Tabla $\mathrm{N}^{\circ} 1$ cada combustible tiene un índice que tiene al Gas Natural Comprimido como combustible de referencia, de tal manera que, por ejemplo, el Diesel $\mathrm{N}^{\circ} 2$ contaminado con 2,500 ppm de azufre (D2-S2500) es 4.04 veces más contaminante que el GNC. 
Tabla $N^{\circ} 1$. Índice de Nocividad de los Combustibles (INC)

\begin{tabular}{|l|l|}
\hline Combustible & Índice (INC) \\
\hline Gas Natural Comprimido (GNC) & 1,00 \\
\hline Gasolinas ecológicas (Hasta 7.8\% de Etanol) & 1,45 \\
\hline Gas licuado de Petróleo (GLP) & 1,45 \\
\hline Gasolinas de 84, 90, 95 y 97 octanos & 1,55 \\
\hline D2-S50 Diesel 2 con 50 ppm S & 2,58 \\
\hline D2-S500 Diesel 2 con 500 ppm S & 2,85 \\
\hline D2-S1500 Diesel 2 con 1,500 ppm S & 3,45 \\
\hline Kerosene & 3,81 \\
\hline D2-S2500 Diesel 2 con 2,500 ppm S & 4,04 \\
\hline Turbo Jet & 4,38 \\
\hline D2-S5000 Diesel 2 con 5,000 ppm S & 5,53 \\
\hline D2-S10000 Diesel 2 con 10,000 ppm S & 8,52 \\
\hline Petróleo Industrial N4 & 9,91 \\
\hline Petróleo Industrial N 5 & 12,99 \\
\hline Carbón & 22,24 \\
\hline Petróleo Industrial N 6 & \\
\hline
\end{tabular}

Una de las aplicaciones del Índice de Nocividad de Combustibles se dio mediante las medidas tributarias de promoción de combustibles limpios incorporadas en el Artículo $3^{\circ}$ de la Ley $\mathrm{N}^{\circ} 28694$ del 22 de marzo de 2006 que señalaba que gradualmente, a partir del $1^{\circ}$ de enero de 2008, se debería determinar el Impuesto Selectivo al Consumo (ISC) a los combustibles, introduciendo el criterio de proporcionalidad al grado de nocividad debido a los contaminantes que éstos contengan. La norma también mandaba que la reestructuración en el ISC se debería realizar de forma gradual hasta el $1^{\circ}$ de enero de 2016 como máximo, fecha en que la tributación que grava los combustibles considerará plenamente el criterio de nocividad. Por lo tanto, en base a este índice se busca promover el consumo de combustibles más limpios mediante la aplicación de un incentivo económico, en este caso el ISC.

A nivel mundial la contaminación de las ciudades regularmente proviene de dos fuentes principales: una móvil, 
originada por el parque automotor, y otra fija, producida por la industria. Largamente la que genera más contaminación es la fuente móvil. Existen otras fuentes de contaminación de las ciudades, pero comparativamente son pequeñas, como es el caso de los humos producidos por las cocinas de los restaurantes y los hogares. Asimismo, a veces entran a tallar otras fuentes, como es el caso de los incendios forestales y las emisiones volcánicas. Por ejemplo, en junio del 2011 las emisiones del volcán Puyehue, ubicado al sur de Chile, llegaron a la ciudad de Buenos Aires obligando incluso al cierre de sus aeropuertos durante varios días.

La contaminación de la ciudad de Lima es similar a la de las grandes ciudades del mundo. Se estima que el $75 \%$ de la contaminación del aire de la capital es causada por los vehículos automotores de todo modelo y antigüedad, por lo que se constituye en el principal problema ambiental de la ciudad. El $25 \%$ restante es generado por las diferentes actividades económicas, especialmente las actividades industriales, debido a las características de los procesos que maneja y a los combustibles que utiliza, en especial las industrias químicas, de harina de pescado y de gases industriales. Un punto importante aquí es la presencia en la gran Lima y Callao de cientos de microindustrias informales de todo tipo, muy contaminantes, entre las que están las ladrilleras y las fundidoras de metales y aleaciones. Asimismo existen en la ciudad otras fuentes, que están en este $25 \%$ restante, que son notables, como es la contaminación originada por la quema de basuras o las emisiones de las chimeneas de los cientos de chifas y pollerias, tan populares en nuestro país.

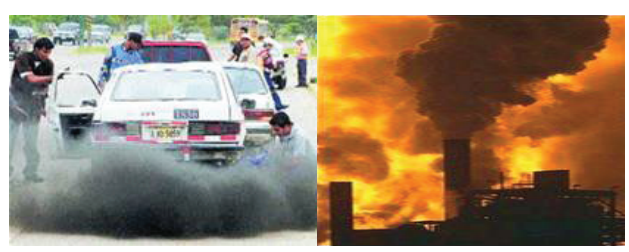

Pero principalmente el problema central en Lima es la contaminación producida por el parque automotor. Este problema se debe a varios factores, que se señalan más adelante, pero el factor principal es la calidad de los combustibles usados. La concurrencia simultánea de estos factores lleva al resultado conocido para la ciudad: una alta contaminación del aire. Por ejemplo, un análisis típico de los gases de combustión que salen por el tubo de escape de una unidad de transporte del tipo combi arroja el siguiente resultado: $92.83 \%$ de dióxido de carbono (CO2), $6.10 \%$ de monóxido de carbono (CO), $0.80 \%$ de compuestos orgánicos volátiles, $0.23 \%$ de óxidos de nitrógeno (NOX), $0.03 \%$ de óxidos de azufre (SOX), y $0.01 \%$ de partículas (carbón, zinc, óxidos, etc.).

En adición a la calidad de los combustibles, otros factores importantes que contribuyen a la contaminación atmosférica de Lima, son los siguientes:

Aumento explosivo del número de vehículos, muchos de ellos importados 
ya usados. Se estima, siguiendo las proyecciones estadísticas del Ministerio de Transportes y Comunicaciones, que en Lima actualmente circulan más de un millón de vehículos automotores diversos. Las estadísticas de las dos últimas décadas indican que por cada vehículo nuevo ingresaban al país de 2 a 3 vehículos usados.

Alta proporción de vehículos antiguos con motores desgastados y/o mal regulados. Durante muchos años han ingresado vehículos de segunda mano con alto recorrido en sus países de origen, lo que ha llevado a que Lima tenga un parque automotor de Lima viejo, del orden de 18 años de antigüedad, en promedio. Adicionalmente, a pesar de la obligatoriedad, muchos no pasan o no aprueban las revisiones técnicas, pero siguen circulando. Por lo tanto es importante no seguir postergando el chatarreo de los taxis que permitiría la renovación de este sector del parque automotor. Es de notar que la importación de vehículos usados finalizó el año 2012.

Calles estrechas y edificaciones altas especialmente en la llamada Lima Cuadrada. Debido a que el Centro Histórico de Lima fue declarado Patrimonio de la Humanidad por la Unesco en 1991 se hace necesario aplicar un plan para evitar su afectación por el parque automotor, que la llena de humos, ruidos, etc. El ejemplo de Cartagena en Colombia es aleccionador.

Poca fluidez del tránsito vehicular, como consecuencia de una deficiente planificación. Aquí ha habido mucha incapacidad de los gobiernos munici- pales y del Ministerio de Transportes, en especial desde la década de 1950, debido a que no se ha preparado a la capital para su crecimiento futuro. Esto se traduce, por ejemplo, en que hasta la fecha solamente tenemos una sola vía, la carretera Panamericana, para atravesar a la ciudad de Norte a Sur y viceversa. Asimismo, en la práctica no tenemos ninguna vía rápida que atraviese la ciudad de Este a Oeste y viceversa, que nos lleve sin interrupciones del Callao a Chosica. Adicionalmente los semáforos no están sincronizados en las calles principales y en las grandes avenidas y las pistas interiores están en mal estado aún en distritos turísticos como Miraflores y Barranco.

Condiciones meteorológicas y topográficas adversas. Por ejemplo, en Lima casi no llueve, lo cual hace que la ciudad no se limpie regularmente y cuando caen las garúas éstas más bien ensucian las pistas y los vehículos de transporte e incluso a los mismos pasajeros. Adicionalmente Lima tiene un viento de baja velocidad y fuerza, originando que la contaminación que se produce en los distritos del sur sea llevada lentamente a los distritos del cono norte, con resultados que cuadruplican la contaminación máxima de partículas aceptada por la Organización Mundial de la Salud.

\section{ACCIONES TOMADAS Y OFERTA DE NUEVOS COMBUSTIBLES}

El inicio de operaciones del Proyecto Camisea en agosto de 2004 trajo a la 
capital dos combustibles ampliamente usados a nivel mundial en automotores: el Gas Natural Vehicular (GNV) y el Gas Licuado de Petróleo (GLP).

El gas natural seco que viene a la costa desde Camisea contiene más del $90 \%$ de metano, cerca de $8 \%$ de etano y cantidades menores de otros gases e hidrocarburos. La empresa Cálidda se encarga de su distribución en la gran Lima y el Callao, comercializándolo en forma de gas residencial, gas comercial, gas vehicular y gas industrial. El gas residencial y comercial se utiliza principalmente en las cocinas y termas; el gas vehicular es el combustible automotor GNV que es adquirido en los servicentros; y el gas industrial es entregado a la industria para usos diversos: combustible, producción de vapor, generación de electricidad en las termoeléctricas de Santa Rosa y Ventanilla, etc. En todos los casos ha sido necesario desarrollar un programa de masificación del uso del gas natural seco, remarcándose sus bondades energéticas y ambientales. Es bueno remarcar que se trata del mismo gas que viene de Camisea y que se vende en la capital con nombres diferentes entre los que está el GNV.

El Gas Licuado de Petróleo (GLP) del proyecto Camisea se obtiene en la unidad de fraccionamiento de Pisco, cerca de la Reserva Nacional de Paracas. En esta unidad se destilan los líquidos del gas natural (LGN) que son traídos a la costa desde Camisea mediante un ducto de líquidos que se desplaza paralelo al ducto de gas. En Pisco se obtienen por destilación los siguientes productos: propano, butano, nafta virgen y diesel. Para obtener el Gas Licuado de Petróleo se mezclan solamente el propano y el butano. Técnicamente el GLP también se obtiene en las unidades de conversión de las refinerías de petróleo. Una unidad de conversión típica es la de craqueo catalítico, presente en las refinerías de Talara y La Pampilla. El GLP automotor se vende exclusivamente en los servicentros. La Tabla $\mathrm{N}^{\circ} 2$ presenta sus características.

Tabla N². Características del Gas Licuado de Petróleo - GLP

\begin{tabular}{|l|l|}
\hline Fórmula & Mezcla comercial de propano y butano \\
\hline Apariencia, color, olor & $\begin{array}{l}\text { Gas comprimido, incoloro, contiene un } \\
\text { odorizante para facilitar detección de fugas }\end{array}$ \\
\hline Gravedad específica $15.6 / 15.6^{\circ} \mathrm{C}$ & $0.54-0.56$ \\
\hline Punto de inflamación, ${ }^{\circ} \mathrm{C}$ & -104 \\
\hline Límites de inflamabilidad, \%V en aire & $2.2-9.5$ \\
\hline Punto de autoignición, ${ }^{\circ} \mathrm{C}$ & 466 \\
\hline Presión de vapor a $37.8^{\circ} \mathrm{C}, \mathrm{psig}$ & $115-208$ \\
\hline Solubilidad en agua & Insoluble \\
\hline Familia química & Hidrocarburos \\
\hline
\end{tabular}


Asimismo tanto las gasolinas como el diesel ya se están vendiendo en Lima mezcladas con biocombustibles y han cambiado de nombre. El marco legal de los biocombustibles en el Perú empezó el 08 de agosto de 2003 en que se emitió la Ley $\mathrm{N}^{\circ} 28054$, Ley de Promoción del Mercado de Biocombustibles, que estableció el marco general para promover el mercado de los biocombustibles sobre la base de la libre competencia y el libre acceso a la actividad económica, para diversificar el mercado de combustibles, fomentar el desarrollo agropecuario y agroindustrial, generar empleo y disminuir la contaminación ambiental. Esta Ley fue reglamentada mediante los Decretos Supremos $N^{\circ}$ 013-2005-EM del 31 de marzo de 2005 y 021-2007-EM del 20 de abril del 2007 que contienen normas para la comercialización de biocombustibles, establecen requisitos para su distribución y hacen referencia a las normas técnicas de calidad. Finalmente los últimos Decretos Supremos que han sido emitidos, en especial el DS 061-2010-EM del 28 de setiembre de 2010, señalan los cronogramas para la comercialización obligatoria en el territorio nacional de gasolinas y diesel 2 conteniendo etanol y biodiesel.

En lo que se refiere a las gasolinas, tal como se indicó anteriormente su venta conteniendo plomo tetraetílico concluyó el 31 de diciembre de 2004. Con la emisión de las normas referidas a biocombustibles el negocio de las gasolinas se fue preparando lentamente para agregarle etanol debido a su alto octanaje y menor emisión de gases de efecto invernadero. Las mezclas gasolina-etanol han sido ampliamente investigadas y aplicadas a nivel mundial, especialmente en Brasil, que tiene el mercado de biocombustibles más desarrollado del planeta. En el Perú se está produciendo bioetanol en el departamento de Piura a partir de caña de azúcar, donde el grupo Romero ha desarrollado nuevas áreas agrícolas en el fundo "Caña Brava" ubicado en Sullana, a la altura de los kilómetros 1,020 a 1,030 de la carretera Panamericana Norte. También se puede obtener bioetanol a partir de sorgo, maíz, yuca, papa, arroz y otros cultivos agrícolas. Los Decretos Supremos 0612010-EM y 024-2011-EM establecieron que el gasohol, es decir la gasolina con un máximo de $7.8 \%$ en volumen de alcohol carburante, debía de iniciar su venta en los departamentos de Piura y Lambayeque el $1^{\circ}$ de abril de 2010 y en Lima Metropolitana y el Callao el 15 de julio de 2011. Este cronograma se ha cumplido, de tal manera que en los servicentros ya no se vende "gasolina" sino "gasohol" de 84, 90, 95 y 97 octanos. Los cuatro gasoholes tienen las mismas especificaciones, variando solo en las especificaciones de octanaje y color. A modo de ejemplo la Tabla $\mathrm{N}^{\circ} 3$ presenta las características de la gasolina de 90 octanos. 
Tabla $\mathbf{N}^{\circ}$ 3. Caracteristicas de la Gasolina de 90 octanos Petroperú

\begin{tabular}{|l|l|}
\hline Fórmula & $\begin{array}{l}\text { Mezcla de Gasolina de } 90 \text { octanos y Alco- } \\
\text { hol Carburante. }\end{array}$ \\
\hline Apariencia, color, olor & $\begin{array}{l}\text { Transparente, amarilla y de olor caracte- } \\
\text { rístico }\end{array}$ \\
\hline Gravedad específica $15.6 / 15.6^{\circ} \mathrm{C}$ & $0.73-0.76$ \\
\hline Punto de inflamación, ${ }^{\circ} \mathrm{C}$ & $<0$ \\
\hline Límites de inflamabilidad, \%V en aire & $1.4-7.6$ \\
\hline Punto de autoignición, ${ }^{\circ} \mathrm{C}$ & 280 \\
\hline Solubilidad en agua & $\begin{array}{l}\text { Hidrocarburo: insoluble en agua. Alcohol } \\
\text { contenido en el Gasohol: soluble en agua }\end{array}$ \\
\hline Familia química & Hidrocarburos y alcohol etílico \\
\hline Composición & $\begin{array}{l}92.2 \% V \text { de Gasolina de } 90 \text { octanos } \\
7.8 \% V \text { de Alcohol carburante }\end{array}$ \\
\hline
\end{tabular}

Con respecto al Diesel como combustible automotor, durante la década anterior se trabajó para reducir su elevado contenido de azufre y para incorporar biodiesel en su preparación. Durante muchos años, mientras en el mundo se consumía un Diesel cada vez más limpio, que llegaba a 50 ppm o menos, en el Perú se mantuvo la comercialización del Diesel con alto contenido de azufre. Así, la Norma Técnica Peruana $N^{\circ} 321.003$ del año 1989, vigente hasta el año 2005, disponía que el contenido máximo de azufre en el Diesel 2 automotor era de 10,000 ppm (1.0 \% en masa). En julio de 2005, debido a las continuas críticas de la opinión pública acerca de la pésima calidad del Diesel, se emitió el Decreto Supremo No 025-2005-EM aprobando el cronograma de reducción progresiva del contenido de azufre en el Diesel $\mathrm{N}^{\circ} 2$ que, en una primera etapa reducía el contenido de azufre a 5,000 ppm a partir del $1^{\circ}$ de setiembre de 2005 y luego reducía dicho contenido a 50 ppm a partir del $1^{\circ}$ de enero de 2010.
Esta norma se ha cumplido solo en su primera etapa. Debido a que las refinerías en el Perú no tienen unidades de desulfurización no es posible producir diesel con 50 ppm o menos. Esto solo será posible cuando entren en operación las nuevas unidades de proceso del proyecto de modernización de la Refinería de Talara, hecho que ocurrirá hacia el año 2018. Paralelamente la Refinería La Pampilla deberá efectuar inversiones similares para modernizar también sus instalaciones.

Con relación al biodiesel, se mencionó anteriormente que durante la década anterior se emitieron diversas normas para promover el mercado de los biocombustibles. El artículo 8 del DS 013-2005-EM de marzo de 2005 ya señalaba que el porcentaje de Biodiesel en el diesel sería de $5.0 \%$ en volumen y lo llamaba Diesel Ecológico $\mathrm{N}^{\circ} 2 \mathrm{E}$; el artículo 9 del mismo DS fijó el $1^{\circ}$ de enero del 2008 como fecha de inicio de la comercialización de este biodiesel en los departamentos de Loreto, Ucayali, San Martín, Ama- 
zonas y Huánuco, y a partir del $1^{\circ}$ de enero del 2010 en todo el país. Esta norma fue modificada por otras que se dieron posteriormente. Así, el DS $\mathrm{N}^{\circ}$ 021-2007-EM definió que el biodiesel es un combustible derivado de recursos renovables tales como aceites vegetales obtenidos del aceite de palma, higuerilla, piñón, soya, colza, girasol y otros, así como de grasas animales. Esta norma especificó que el biodiesel puro se llamaría Biodiesel B100 y que podía mezclarse con el diesel en tres porcentajes: $2 \% \mathrm{~V}$ de B100 y $98 \% \mathrm{~V}$ de diesel (el producto se llamaba Diesel B2), $5 \% \mathrm{~V}$ de B100 y $95 \% \mathrm{~V}$ de diesel (el producto se llamaba Diesel B5) y $20 \% \mathrm{~V}$ de B100 y $80 \% \mathrm{~V}$ de diesel (el producto se llamaba Diesel B20). Este mismo DS No 021-2007-EM también señalaba que a partir del $1^{\circ}$ de enero de 2009 la venta de Diesel B2 sería obligatoria en todo el país en reemplazo del Diesel $\mathrm{N}^{\circ} 2$ y que a partir del $1^{\circ}$ de enero del 2011 la venta de Diesel B5 sería obligatoria en todo el país en reemplazo del Diesel B2. Este cronograma se ha cumplido y, por lo tanto, a partir del $1^{\circ}$ de enero de 2011 solo se comercializa el Diesel B5. La Tabla $\mathrm{N}^{\circ} 4$ presenta las características actuales de este combustible.

Tabla N4. Caracteristicas del Diesel B5

\begin{tabular}{|l|l|}
\hline Fórmula & Mezcla de diesel con biodiesel B100 \\
\hline Apariencia, color, olor & $\begin{array}{l}\text { Líquido claro y brillante, color visual ám- } \\
\text { bar y olor característico }\end{array}$ \\
\hline Gravedad específica $15.6 / 15.6^{\circ} \mathrm{C}$ & $0.82-0.87$ \\
\hline Punto de inflamación, ${ }^{\circ} \mathrm{C}$ & 52 \\
\hline Límites de inflamabilidad, \%V en aire & $1.3-6.0$ \\
\hline Punto de autoignición, ${ }^{\circ} \mathrm{C}$ & 257 \\
\hline Solubilidad en agua & Insignificante \\
\hline Familia química & Hidrocarburos y ésteres monoalquílicos \\
\hline Composición & $\begin{array}{l}95.0 \% \mathrm{~V} \text { de Diesel } 2 \\
5.0 \% \mathrm{~V} \text { de Biodiesel B100 puro }\end{array}$ \\
\hline
\end{tabular}

\section{CONCLUSIONES}

- El conjunto de combustibles que se comercializa actualmente es totalmente diferente al grupo de combustibles que se comercializaba en Lima Metropolitana y el Callao hasta la década pasada.

- A fines del siglo pasado se comercializaba en Lima Metropolitana y el Callao cuatro gasolinas: gasolina 84 con plomo, gasolina $90 \mathrm{sin}$ plomo, gasolina 95 con plomo y gasolina 97 sin plomo. Asimismo el parque automotor se abastecía de Diesel $\mathrm{N}^{\circ} 2$ con diez mil ppm de azufre. La mala calidad de estos combustibles, que afectaba el medio ambiente de la ciudad y la salud de la población, originó continuas críticas de especialistas y de la opinión pública que llevó a una lenta mejora en la calidad de estos combustibles. 
- Desde el $1^{\circ}$ de enero de 2005 ninguna gasolina en el Perú contiene plomo.

- Desde el año 2011 se ha dejado de vender gasolinas en Lima Metropolitana y el Callao. En su lugar se expende gasohol de 84, 90, 95 y 97 octanos. El gasohol es una mezcla de $92.2 \%$ en volumen de gasolina con $7.8 \%$ en volumen de alcohol etílico (etanol) obtenido actualmente de la caña de azúcar.

- Desde el $1^{\circ}$ de setiembre de 2005 se redujo el contenido de azufre en el Diesel a 5,000 ppm. Está pendiente reducir aún más este contenido a $50 \mathrm{ppm}$, lo que se conseguirá cuando termine el proyecto de modernización de la Refinería de Talara, que incluye nuevas unidades de desulfurización, así como de Refinería La Pampilla.

- El Diesel conteniendo biodiesel empezó a comercializarse desde el año 2009. Del $1^{\circ}$ de enero de 2009 al 31 de diciembre de 2010 se vendió Diesel B2, que tenía $98 \%$ en volumen de diesel y $2 \%$ en volumen de biodiesel. A partir del $1^{\circ}$ de enero de 2011 solamente se vende el Diesel B5. Este biocombustible tiene $95 \%$ en volumen de diesel, $5 \%$ en volumen de biodiesel.

- En adición a los gasoholes y al biodiesel, nuestro parque automotor también tiene a su disposición Gas Natural Vehicular (GNV) y Gas Licuado de Petróleo (GLP). El GNV proviene de Camisea y el GLP es obtenido en la planta de fraccionamiento de Paracas y en las uni- dades de craqueo catalítico de las refinerias de Talara y La Pampilla.

\section{PROPUESTAS A FUTURO}

- Continuar profundizando el cambio de la matriz energética de combustibles en el país, que debe hacerse efectivo con mayores descubrimientos de gas natural y/o petróleo crudo, labor a cargo de la empresa Perúpetro que, en representación del Estado, promueve la inversión en actividades de exploración y explotación de hidrocarburos en el Perú.

- El gobierno central debe asegurar la reserva del gas natural del Lote 88 para el mercado interno. De esta manera se asegurará también el suministro de GNV y GLP para el parque automotor y para otras actividades económicas no discutidas en este artículo como la petroquímica, fertilizantes, gas para el sur del país, gasoductos secundarios para la región central y norte del país, etc.

- El Ministerio de Energía y Minas debe evaluar la conveniencia de programar con suficiente anticipación la comercialización de Diesel B20, especificado en el DS N $\mathrm{N}^{\circ} 021$ 2007-EM del año 2007. Este combustible tendrá $20 \%$ en volumen del biodiesel puro B100 y $80 \%$ en volumen del diesel común. De esta manera el sistema de producción, transporte y comercialización del Diesel B20 podrá prepararse con tiempo para atender adecuadamente esta disposición. 
- El gobierno central debe apoyar ampliamente y acelerar la ejecución del proyecto de Modernización de la Refinería de Talara que tiene como objetivo incrementar la capacidad de la refineria en $50 \%$, producir destilados de gasolina y diesel con menos de 50 ppm de azufre y procesar crudos pesados que son más económicos para la producción de combustibles livianos de mayor valor comercial.

- El gobierno central debe hacer uso del Índice de Nocividad de Combustibles para definir el precio de venta de los combustibles, aplicando apropiadamente el impuesto selectivo al consumo, de tal manera que los combustibles más nocivos al medio ambiente y a la salud paguen los mayores impuestos.
- El gobierno central debe evaluar adecuadamente otros proyectos para monitorear y reducir la contaminación en la gran Lima y el Ca1lao. Entre ellos tenemos: (1) Dotar a toda la ciudad de un amplio sistema de control de la contaminación ambiental con equipos nuevos; hoy solamente existe la antigua Estación CONACO en el centro de Lima; (2) Implantar un programa de Alerta Ambiental, en base al monitoreo de la calidad del aire, tal como lo tienen ciudades muy contaminadas como México DF; (3) Acelerar el proyecto de chatarreo de autos concebido inicialmente para taxis, y no continuar postergándolo; (4) Revisar el Índice de Nocividad de Combustibles; el actual data del año 2005.

\section{REFERENCIAS BIBLIOGRÁFICAS}

Blumberg, Katherine; Walsh, Michael \& Pera, Charlotte, (2003). "Gasolina y diesel de bajo azufre: la clave para reducir las emisiones vehiculares". España: Fundación Hewlett.

Cala, David. (2007). "Bioalcohol para las gasolinas y biodisel para el diesel". Revista Petróleo Internacional, Miami.

Consejo Nacional del Ambiente, (2005). Decreto del Consejo Directivo $\mathrm{N}^{\circ}$ 018-2005-CONAM/CD: "Índice de Nocividad de Combustibles para el periodo 2005-2006”. Diario Oficial El Peruano, Lima.

Congreso de la República. (2003). Ley 28054: "Ley de promoción del mercado de biocombustibles”. Diario Oficial El Peruano.

Congreso de la República. (2006). Ley No 28694: "Ley que regula el contenido de azufre en el Diesel”. Diario Oficial El Peruano, Lima.

Dalmasso, Juan Pablo (2006). "Oro verde - biocombustibles". Revista América Economía, Lima. 
Defensoria del Pueblo. (2008). "La calidad del aire en Lima y su impacto en la salud y la vida de sus habitantes". Informe Defensorial $\mathrm{N}^{\circ} 116$. Lima.

Ministerio de Energía y Minas. (2005). Decreto Supremo No 013-2005-EM: "Reglamento de la Ley promoción del mercado de biocombustibles". Diario Oficial El Peruano.

Ministerio de Energía y Minas. (2005). Decreto Supremo No 025-2005-EM: “Cronograma de reducción progresiva de azufre en el diesel 1 y 2". Diario Oficial El Peruano, Lima.

Ministerio de Energía y Minas. (2007). Decreto Supremo No 021-2007-EM: "Reglamento para la comercialización de biocombustibles". Diario Oficial El Peruano, Lima.

Ministerio de Energía y Minas. (2010). Decreto Supremo No 061-2010-EM: "Modifican Reglamento para la comercialización de biocombustibles". Diario Oficial El Peruano, Lima.

Ministerio de Transportes y Comunicaciones. (1998). Decreto Supremo No 019-98-MTC: "Eliminan del mercado la oferta de gasolina 95 con plomo y reducen el límite máximo de contenido de plomo en la gasolina 84". Diario Oficial E1 Peruano, Lima.

Petroperú. (2007). "Hoja de datos de seguridad del GLP”. Lima.

Petroperú. (2010). "Hojas de datos de seguridad de los gasoholes de 84, 90, 95 y 97 octanos". Lima.

Petroperú. (2011). "Hoja de datos de seguridad del Diesel D5". Lima.

Regalado, Antonio \& Radowitz, Bernd. (2005). “Con la mira en Japón, Petrobras entra fuerte al negocio del etanol”. Diario El Comercio, Lima.

Servicio Nacional de Meteorología e Hidrología. (2011). "Vigilancia de la calidad del aire en la zona metropolitana de Lima y Callao". Boletín mensual. SENAMHI, Callao.

Silva, José \& Montoya, Zarela. (2005). “Análisis del comportamiento estacional de los CSS con las condiciones meteorológicas predominantes en la zona metropolitana de Lima-Callao, durante el año 2004”. SENAMHI, Callao.

Unger, Tomás. (2009). "El biodiesel y el alcohol". Diario El Comercio, Lima.

Unger, Tomás. (2007). "Biocombustibles y crisis energética”. Diario El Comercio, Lima. 